\title{
Role of NS1 Antigen in Early Detection of Dengue during Epidemics
}

\author{
V.R. Vidhya* and N. Palaniappan
}

Sree Mookambika Instiute of Medical Sciences, Kulasekaram, Kanyakumari District, India

*Corresponding author

\section{A B S T R A C T}

\begin{tabular}{|l|}
\hline Ke y w o r d s \\
Dengue, \\
Aedes aegypti \\
NS1 Antigen \\
\hline Article Info \\
\hline $\begin{array}{l}\text { Accepted: } \\
\text { 04 March } 2019 \\
\text { Available Online: } \\
\text { 10 April } 2019\end{array}$ \\
\hline
\end{tabular}

\begin{abstract}
Dengue has become a major international public health problem due to human morbidity and mortality it causes. Dengue NS1 antigen has been detected in the serum of DEN virus infected patients as early as one day of onset of symptoms. In this study the potential use of dengue NS1 antigen and in early dengue diagnosis has been analysed. The study group consists of all febrile patients with fever of duration less than 5 days attending the medical OPD for a period of one year from July 2013. The blood sample was subjected to an immunochromatographic test for NS1 antigen, IgM and IgG antibodies. This was followed by ELISA for NS1 antigen, IgM and IgG antibodies. Out of 176 samples tested, 57 (32\%) were positive for NS1 antigen by immunochromatographic test. The 57 samples which were found to be positive by NS1 ELISA were also found to be positive by immunochromatographic test. NS1 antigen detection is a useful tool in the early diagnosis of dengue infection and deserves inclusion in the diagnostic algorithm for suspected dengue fever cases. The results of NS1 antigen immunochromatographic test are comparable to that of NS1 antigen ELISA.
\end{abstract}

\section{Introduction}

Dengue virus belongs to Flaviviridae family and the virus could be found in tropical and sub tropical regions worldwide. Dengue fever is an acute, potentially fatal viral infection that is endemic throughout India $^{1}$ Transmission of dengue virus in Asia is maintained by a native mosquito species, the Aedes albopticus. When the virus was introduced into an area that had become infested with the Aedes aegypti mosquito, dengue epidemics occurred ${ }^{2}$.

According to WHO estimates dengue viral infections are responsible for hundred million cases of dengue infections annually and more than five lakh cases of dengue haemorrhagic fever ${ }^{5}$. The main factors which had made dengue infections a global health problem is unprecedented global population growth, unplanned and uncontrolled urbanisation and deterioration in water, sewer and waste management systems ${ }^{4}$. The emerging dengue viral infections is of great threat to the mankind as there is no specific medication available nor there is any vaccine available against dengue virus. ${ }^{6}$

Dengue virus consists of four distinct serotypes based on their antigens, namely DENV1, DENV2, DENV3 and DENV4. Dengue virus consists of a positive stranded, encapsulated RNA as their genetic material which is $11 \mathrm{~kb}$ long and has a single open reading frame (ORF) which codes for a single 
polypeptide. This polypeptide consists of three structural proteins and seven nonstructural proteins. The structural proteins are named as core protein $\mathrm{C}$, membrane protein $\mathrm{M}$ and envelope protein $\mathrm{E}$. The seven non structural proteins are NS1, NS2A, NS2B, NS3, NS4A, NS4B and NS5. ${ }^{7}$

NS1 protein is a 50 kilodalton glycoprotein (353 or 354 amino acids), which is expressed on the surface of cells infected with dengue virus. It is produced in both membrane associated and secreted forms.. It may be detected in the blood from the first day of fever up to the fifth day of fever which is the critical period of illness preceeding defervescence which generally occurs by the fifth day ${ }^{10,}{ }^{11}$. Therefore commercial diagnostic kits have utilised virus coded NS1 antigen as a basis for early detection of dengue viral infections ${ }^{12}$.

The antibody production following Dengue viral infection consists of $\operatorname{IgM}$ and $\operatorname{IgG}$ antibodies which are produced against the viral envelope proteins. The type of antibody production may depend on whether an individual has a primary dengue viral infection or a secondary dengue viral infection $^{11}$. The first immunoglobulin isotype to appear in primary dengue infection is IgM. IgM antibodies may be detectable approximately 3 to 5 days after the onset of fever and then its levels increases rapidly and peaks for 2 weeks after the onset of fever. However IgG immunoglobulins are detectable at low titres by the end first week of illness and then it rises slowly. The level of $\mathrm{IgG}$ antibodies decline slowly over the next 3 to 6 months ${ }^{8}$.

Since, Kulasekharam is endemic to dengue, detection of early cases by antibody method does not give a correct number of dengue cases. This is because people in this area will have either serum IgG or IgM antibodies.
Therefore in this study the potential use of dengue NS1 antigen in early dengue diagnosis is analysed and compared to the current antibody methods available in our laboratory.

\section{Materials and Methods}

The study group consists of all febrile patients with fever of duration less than 5 days attending the medical OPD for a period of one year from July 2013. The study was done after receiving permission from institutional ethical and research committee. A total of 176 blood samples from fever patients were received in the microbiology lab of central lab in our institution. The samples were subjected to an immune chromatographic test (card test) for NS1 antigen, $\operatorname{IgM}$ and $\operatorname{IgG}$ antibodies. This was followed by ELISA (Enzyme Linked Immunosorbent Assay) for detection of NS1, IgM and IgG antibodies of dengue virus. The manufacturer instructions were strictly followed.

\section{Results and Discussion}

The study was done using multi variant regression analysis with dependent binary variables. This model also assumes that $Z$ is linearly related to the predictors. ${ }^{114,115}$

$Z_{i}=b_{0}+b_{1} x_{\mathrm{i} 1}+b_{2} x_{\mathrm{i} 2}+b_{p} x_{\mathrm{ip}}$

Where

$$
\begin{aligned}
& x_{\mathrm{ij}} \text { is the } j^{\text {th }} \text { predictor for the } i^{\text {th }} \text { case } \\
& \mathrm{b}_{\mathrm{j}} \text { is the } j^{\text {th }} \text { coefficient } \\
& p \text { is the number of predictors }
\end{aligned}
$$

$\mathrm{Z}$ is a binary variable which takes value 1 for positive sample and zero for negative sample.

From figure 2 it could be shown that $57 \%$ of the total sample were positive for one of the dengue ELISA (NS1 antigen, IgM/IgG antibody). Out of the $57 \%, 32 \%$ were positive for NS1 ELISA, 21\% for IgM ELISA and 4\% for IgG ELISA (Fig. 1). 
In figure $3,36 \%$ of the samples were positive for immunochromatographic test, out of which $32 \%$ were positive for NS1 antigen and only $4 \%$ were positive for dengue antibodies.

Table.1 Comparison of efficacy of various dengue specific seromarkers in the diagnosis of dengue infection

\begin{tabular}{|l|l|l|}
\hline $\begin{array}{l}\text { DENGUE } \\
\text { SEROMARKERS }\end{array}$ & CARD TEST POSITIVE & ELISA POSITIVE \\
\hline NS1antigen & $57(32 \%)$ & $57(32 \%)$ \\
\hline IgM antibody & $7(4 \%)$ & $37(21 \%)$ \\
\hline IgG antibody & 0 & $7(4 \%)$ \\
\hline
\end{tabular}

Table.2 Comparison of platelet counts with various permutations and combinations of dengue parameters

\begin{tabular}{|l|l|l|l|}
\hline $\begin{array}{l}\text { SEROLOGICAL } \\
\text { MARKERS }\end{array}$ & TOTAL & $\begin{array}{l}\text { PLATELET COUNT } \quad \text { LESS } \\
\text { THAN 100,000ML }\end{array}$ & PERCENTAGE \\
\hline NS1 antigen & 57 & 42 & $74 \%$ \\
\hline IgM antibody & 37 & 18 & $48 \%$ \\
\hline IgG antibody & 7 & 2 & $28 \%$ \\
\hline
\end{tabular}

Fig.1 Bar diagram comparing NSI antigen ELISA test and NSI antigen card test

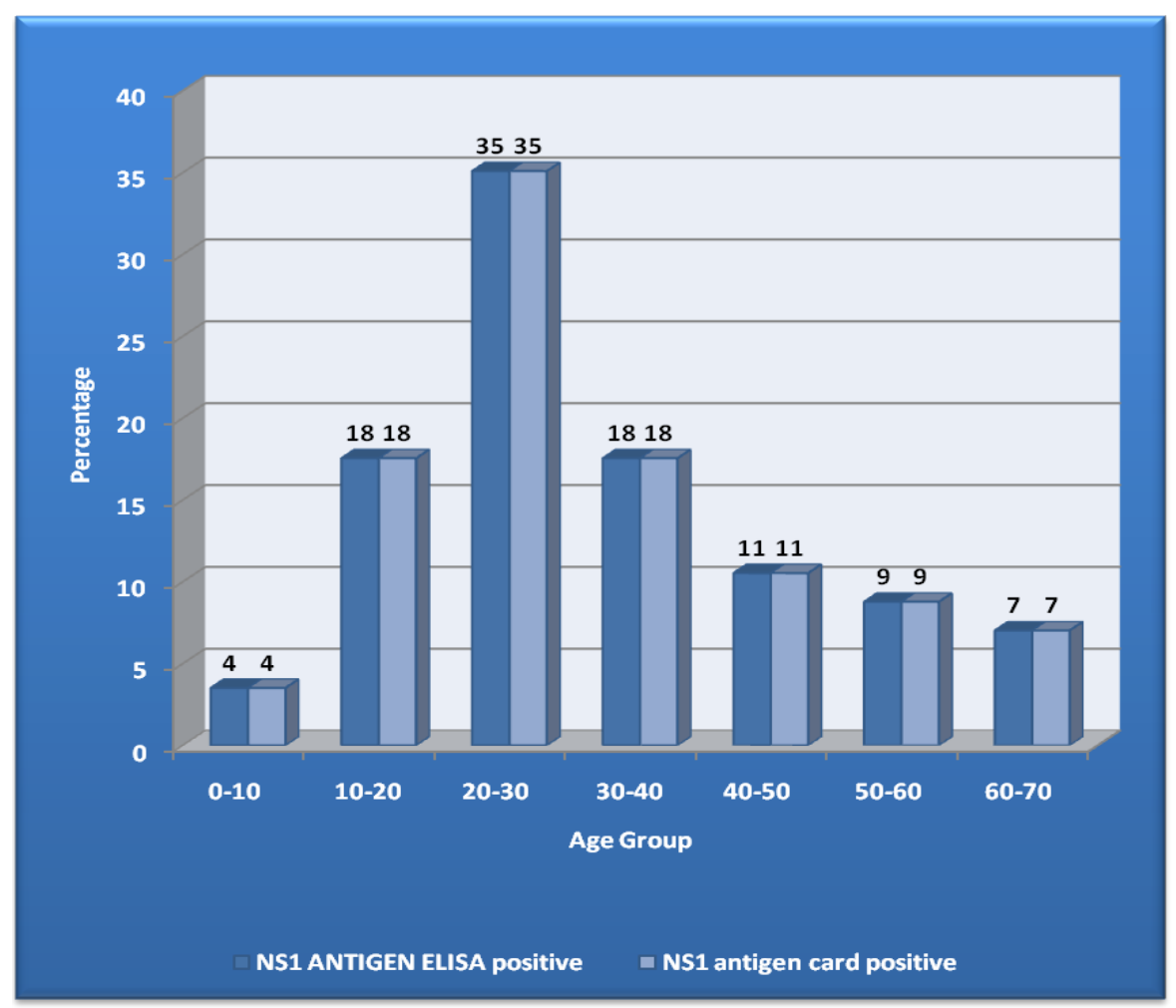


Fig.2 Pie Chart for ELISA Test

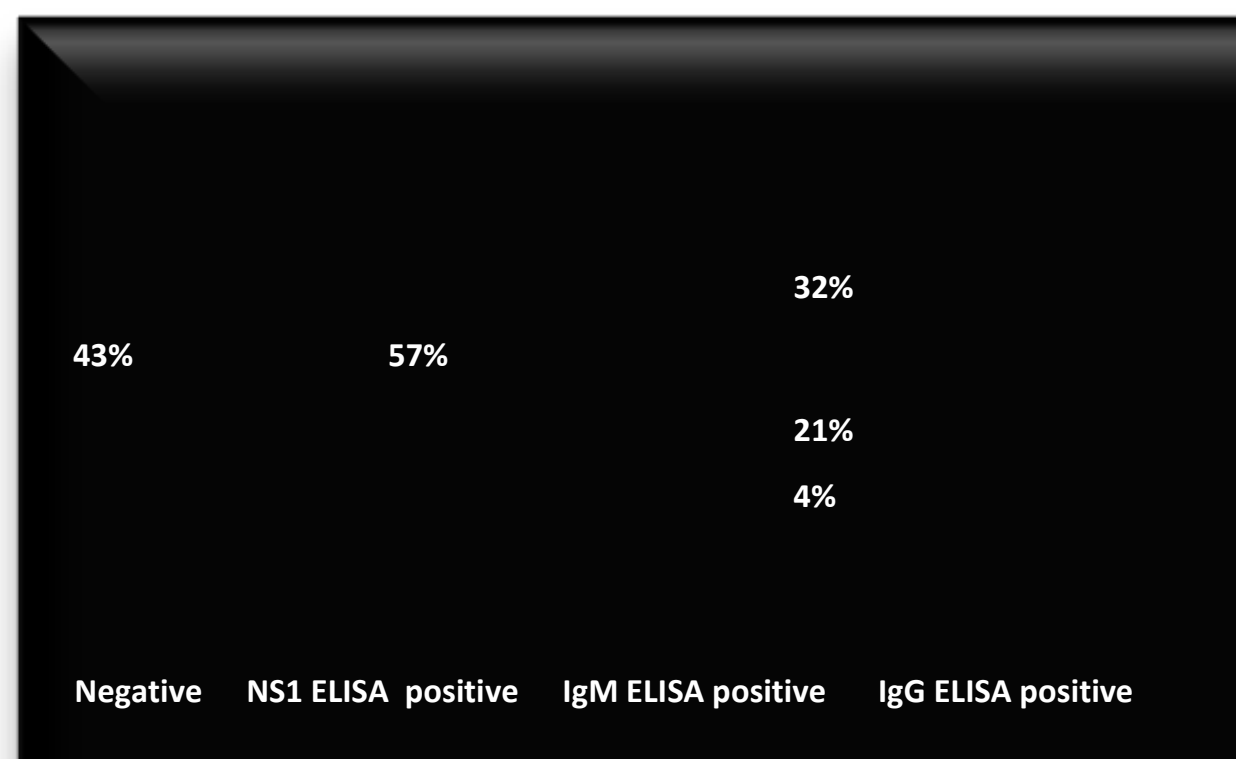

Fig.3 Pie chart for, ICT (Card Test)

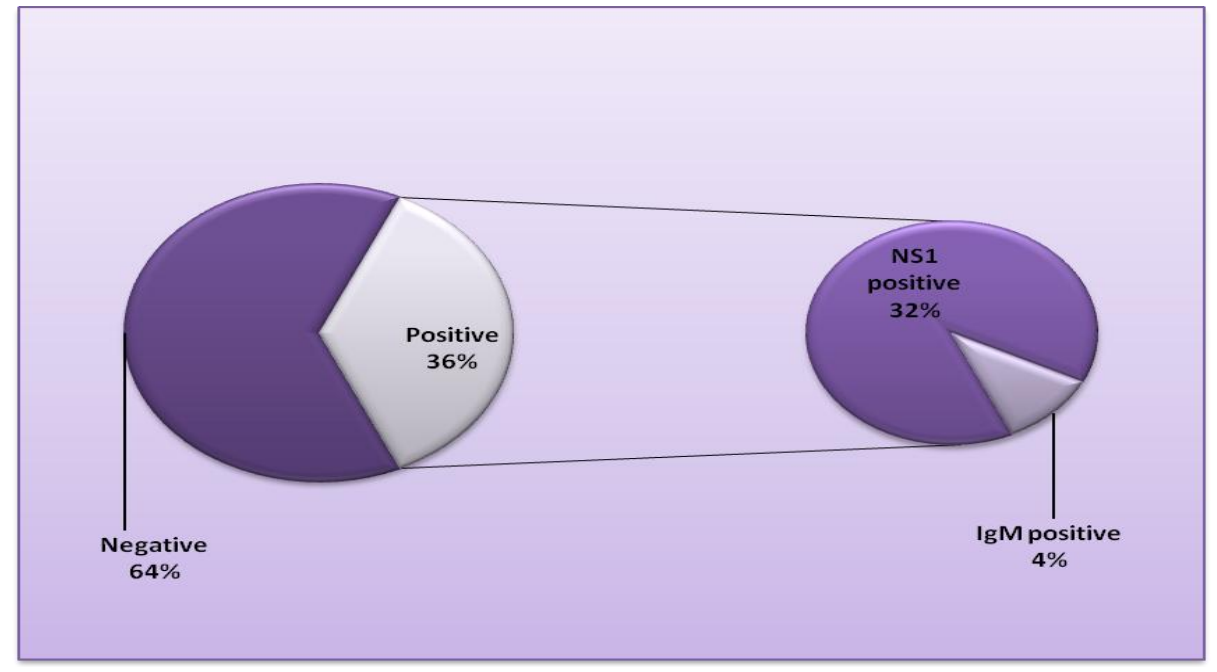

Dengue fever is an important mosquito borne viral disease of humans. The disease is now present in more than hundred countries in the world. The methods for detection of the virus (by cell culture, immunofluorescence), detection of virus antigen (by enyzme linked immunosorbent assay, ELISA), detection of anti-dengue virus antibody [by haemagglutination Inhibition, (HI), complement fixation (CF), neutralisation tests, ELISA ] and detection of viral nucleic acid (by reverse transcriptase polymerase chain reaction). However for confirmed dengue diagnosis dengue virus should be identified by isolation or nucleic acid detection or there should be a four fold rise in antibody titre in paired sera in patients presenting with signs and symptoms consistent with dengue virus infection ${ }^{9}$. 
The distribution of various specific dengue parameters is shown in table 1. Considering a very high sensitivity of NS1 it can be stated that, the diagnosis in $32 \%$ of cases would have been missed if NS1 was not included in the test panel. Kulkarni (2011), Shrivastva (2011), Datta (2010) have shown that NS1 was positive in 95 out of 320 cases (30\%), 15 out of 91 cases $(16 \%)$ and 140 out of 600 cases $(23 \%)$ respectively in their studies ${ }^{6}$. From the above it can be said that a large number of cases would be missed if NS1 antigen is not included in the test panel for dengue virus infection detection. Those who are exclusively NS1 positives could be offered appropriate supportive therapy, thus avoiding any irrational use of antibiotics. The dengue specific antibodies appear only by third to fifth day of fever in primary dengue infection $^{10}$. Even in most secondary infections, both $\operatorname{IgM}$ and $\operatorname{IgG}$ type antibodies cannot be recorded before the third day. Moreover the utility of antibody relies mainly on the rising titres especially in endemic areas. The seromarker NS1 antigen which is a highly sensitive dengue marker for dengue infections is detected from the first day of fever $^{11}$. Among the 57 NS1 antigen positive cases $28(49 \%)$ were detected on the second day of fever

Following the initial infection, the dengue virus replicates to high titres in the blood before the patient becoming symptomatic enough to go to a physician ${ }^{12}$. During the viraemic phase of dengue infection, NS1 antigen are produced during virus replication in either membrane associated or secreted forms. Soluble NS1 circulates in the serum of patients of these patients and thus provides an excellent tool for diagnosis of acute dengue infections ${ }^{13}$.

In this study ELISA is taken as the gold standard for serological testing of dengue fever. NS1antigen was positive in 57 /
$176(32 \%)$ cases by immunochromatographic test (Fig. 3). These 57 cases (32\%) were also found to be positive for ELISA (Fig. 2) and those cases which were found to be negative by card test were found to be negative by ELISA. Thus it could be shown that the rapid immunochromatographic test used in this study is equally sensitive and specific to ELISA. Although virus isolation is considered to be the gold standard for laboratory diagnosis of dengue viral infections, it involves large labour and requires atleast 6-10 days for virus to replicate ${ }^{1,15}$. On the other hand RT-PCR is expensive and require sophisticated infrastructure, technically skilled manpower which are not available in most of the hospitals ${ }^{14}$. With increasing incidence of dengue infections, the early diagnostic confirmation is required for timely intervention and disease control. Hence, diagnosis of dengue disease during the acute phase should be the priority and is a public health concern. In a developing country like India where resources are minimal a Rapid immunochromatographic test method could be useful as a point of care test to provide early diagnosis for timely intervention ${ }^{16}$.

In the study 7 cases (4\%) out of 176 were IgM positive by immunochromatographic test (Table 1). By ELISA method a total of 37 (21\%) out of 176 cases were positive for IgM. Additionally 30 samples were detected as IgM positive by ELISA method (Fig. 2). Here we can see that these patients may have presented in the later stage of the primary infection by dengue virus. In addition, $\operatorname{IgM}$ antibody response varies considerably among individuals due to host humoral immune response. By immunochromatographic test, no samples was found to be positive by IgG (Fig. 3, Tab1e) but by ELISA 7 (4\%) were detected as IgG positive (Fig. 2, Table 1). So we can see that these seven IgG positive patients may have presented during their secondary infection. A high level of IgG is an 
indicator of infection or it represents endemicity $^{17}$. A high degree of cross reactivity is mostly observed in flavi virus infection because the flavi viruses share common group epitopes particularly on the envelope (E) protein $^{18}$. The dengue serological tests become more difficult, because dengue antibodies are cross reactive with other flavi viruses such as West Nile virus (WNV), Japanese encephalitis virus (JEV) and Yellow fever virus (YFV). Moorthy (2009) has evaluvated a rapid immunochromatographic device for detection of $\mathrm{IgM}$ and $\mathrm{IgG}$ antibodies to dengue viruses done in tertiary care hospital.

Accuracy indices for IgM and IgG detection respectively were positive predictive value of $61 \%$ and $72 \%$ and negative predictive value of $89 \%$ and $83 \%$ respectively. it was concluded that the device performs poorly in the detection of $\operatorname{IgM}$ and $\operatorname{IgG}$ antibodies to dengue virus and is not recommended for use as a stand-alone diagnostic test ${ }^{19}$.

In this study an attempt has been made to find out the association of dengue serological marker with thrombocytopenia. Platelet count less than $100,00 / \mathrm{ml}$ is taken as thrombocytopenia. Out of the 176 cases 123 cases $(70 \%)$ has shown thrombocytopenia. The comparison of platelet counts with dengue specific parameters is shown in table 2. Out of the 57 cases positive for NS1 antigen, thrombocytopenia was evident in 42 cases (74\%). In contrast, when IgM and IgG antibodies were compared only 20 (45\%) out of 44 cases had thrombocytopenia. Therefore early viraemic phase coincides with high NS1 antigen levels in the blood which in turn is related to thrombocytopenia ${ }^{20}$.

From figure 2 it could be shown that $57 \%$ of the total sample was positive for one of the dengue ELISA (NS1 antigen, IgM/IgG antibody). Out of the $57 \%, 32 \%$ were positive for NS1 ELISA, $21 \%$ for IgM ELISA and $4 \%$ for IgG ELISA.

Association of thrombocytopenia with NS1 was found to be $74 \%$ while association of thrombocytopenia with $\operatorname{IgM}$ and $\operatorname{IgG}$ was found to be $50 \%$ (Table 2). The mechanism of dengue related thrombocytopenia and coagulopathy is quite complex. So viraemia correlates with NS1 positivity and thrombocytopenia. It would involve platelet activation, pro-coagulant and anticoagulant arms of the coagulation system, complement, cytokines, and endothelial cells. Moreover symptomatic thrombocytopenia would require platelet transfusion though platelet counts might not correlate well with clinical bleeding $^{21}$.

This study is conducted in a tertiary care teaching hospital which lack a viral culture set up. Therefore applying gold standard tests in the studies related to viral infection is not practical for diagnosis and treatment purpose. The precise day of fever at the time of conducting the test could not be obtained in a large number of cases. NS1 antigen was positive in $32 \%$ of cases by both tests. It indicates that if the samples were taken on the first day of fever more number of cases would have been picked up by NS1 antigen ${ }^{22}$. It is shown that the titres of NS1 represents the viral load and the viral load is directly proportional to the complications. Conventional ELISA require at least 4 hours whereas rapid immunochromatographic test requires twenty minutes, which will be helpful in initiating the treatment and minimizing the complications and mortality of dengue ${ }^{20}$. But the lack of conformity in the evaluation of Dengue rapid diagnostic tests (RDTs) remains a problem and a standardised approach must be performed when performing diagnostic assessment. Moreover studies have demonstrated that Rapid diagnostic test cannot reliably differentiate the different dengue infection states (primary or secondary dengue infection). The manufacturers of the kit used in this study 
allows the use of serum, plasma or whole blood for use in dengue rapid diagnostic tests ${ }^{1}$. Unfortunately there is little evidence that all sample types perform equally well. The effect of anticoagulants and whole blood on RDT performance and ease of reading also require examination in a field setting ${ }^{11}$.

In conclusion, this study suggests that NS1 assays deserve inclusion in the diagnostic evaluation of dengue patients, but with due consideration for limitations in patients who present late in their illness or have a concomitant humoral immune response.

\section{References}

1. Monique da Rocha Queiroz Lima, Rita Maria Riberio Nogueria, Herman Goncalves Schatzmayr, Flavia Barreto et al., Comparison of Three Commercially Available Dengue NS1 Antigen Capture Assays for Acute Diagnosis of Dengue in Brazil. PLOS Negl Trop Dis 2010;4(7):1-7.

2. Datta S, Wattal C. Dengue NS1 antigen detection: A useful tool in early diagnosis of dengue virus infection. Indian J Med Microbiol 2010;28 (2):107-10.

3. A special issue on Dengue ENVIS News Letter Volume 4, Issue 1, Nov 2007;4(1):1-16.

4. Kwoon-Yong Pok, Yee-Ling Lai, Joshua Sng, Lee-Ching Ng. Evaluvation of Nonstructural 1 Antigen Assays for the Diagnosis and Surveillance of Dengue in Singapore. Vector-borne and Zoonotic Dis J 2010;10(10):1009-16.

5. Scott R Fry, Michelle Meyer, Mathew G Semple, Cameron P Simmons, Shamala Devi Sekaran, Johny X. Huang, Catriona McElnea, Chang-Yi Huang, Andrea Valks, Paul R.Young, Mathew A et al. The Diagnostic Sensitivity of Dengue Rapid Test Assays Is Significantly
Enhanced by Using aCombined Antigen and Antibody Testing Approach. PLOS Negl Trop Dis 2011;5(6):1199-213.

6. Kulkarni RD, Patil SS, Ajantha GS, Upadhya AK, Kalabhavi AS, Shubhada RM, Shetty PC, Jain PA et al. Association of platelet count and serological markers of dengue infection - importance of NS1 antigen. Indian J Med Microbiol 2011;29(4):359-62.

7. Fauziah Md Kassim, Nur Izati, Rogayah TAR, Mohd Apandi, Zainah Saat et al. Use of Dengue NS1 antigen for early diagnosis of dengue virus infection. SouthEast Asian J Trop Med Public Health 2011;42(3):562-9.

8. Shrivastava A, Dash PK, Tripathi NK, Sahni AK, Gopalan N, Lakshmana Rao. Evaluation of a commercial Dengue NS1 Enzyme linked immunosorbent assay for diagnosis of dengue infection. Indian $\mathbf{J}$ Med Microbiol 2011;29(1):51-5.

9. Nivedita Gupta, Sakshi Srivstava, Amita Jain, Umesh C Srivastava. Dengue in India. Indian J Med Res 2012; 136(9):373-90.

10. Holly R Hughes, Wayne D Crill, Gwong-Jen JChang. Manipulation of immunodominant dengue virus $\mathrm{E}$ protein epitopes reduces potential antibody dependent enhancement. Virol J 2012; 23 (4):123-9.

11. Jayaratne SD, Vajini Atukorale, Laksiri Gomes, Thashi Chang, Tharindu Wijesinghe, Sachie Fernando, Graham S Ogg, Gathsaurie et al. Evaluation of the WHO revised criteria for classification of clinical disease severity in acute adult dengue infection. BMC research notes 2012;23(7):2-9.

12. Aryati Aryati, Trimarsanto, Benediktus Yohan, Pushpa Wardhani, Sukmal Fahri, Tedjo Sasmono et al. Performance of commercial dengue NS1 ELISA and molecular analysis of NS1 gene of dengue viruses obtained during 
surveillance in Indonesia. BMC Med Central 2013;13(6):1471-81.

13. Anantha Narayanan and Paniker's Text book of Microbiology. $9^{\text {th }}$ Edn. 519,692.

14. Tewari KN, Tuli NR, Devagun SC. Clinical profile of dengue fever and the use of platelets in four tertiary level hospitals of Delhi in the year 2009. J Indian Academy Clin Med 2013;14(1):112.

15. Saurabh G Agarwal, Atul R Rudikar, Avinash Laghawe, Apoorva Tripathi. Importace of platelet count and serological markers in Diagnosing Dengue Infections with special reference to NS1 antigen. India $\mathbf{J}$ Med Microbiol 2013; 34(8):43-8.

16. Muhammed Zaman Khan Assir, Umair Kamaran, Hafiz IjasAhmad, Sadia Bashir. Effectiveness of platelet transfusion in Dengue fever: A Randomised Controlled Trial. Transfus Med Hemother 2013; 40: 362-8.

17. Vivaldo G. Da Costa, Ariany C Marques-Silva, Marcos L. Morell. A meta-analysis of the diagnostic Accuracy of two commercial NS1 antigen tests for early dengue virus detection. PLOS Negl Trop Dis 2014;9(4):9465-71.

18. Kwissa M, Nayaka HL, Onalmoon N, Wrammer J, Villinger F, Perng GC, Yoksan S, Pattanapanyasat K, Chokephaibulkit K, Ahmed R, Pulendran et al. Dengue virus infection induces expansion of a CD 14 (+) CD16(+) monocyte population that stimulates plasma blast differentiation. Cell Host Microbe 2014;16(7):115-27.

19. Moorthy, Chandy, Selvaraj K, Abraham AM. Evaluvation of a rapid immunochromatographic device for the detection of IgM and IgG antibodies to dengue virus in a tertiary care hospital in South India. Indian J Med Microbiol 2009;27(3):254-6.

20. Silva MM, Dias AC, Cordeiro MT,Marques E Jr, Goulart MQ, Dutra et al. A thiophene-modified screen printed electrode for detection of dengue virus NS1 protein. Pub Med Commons home 2014; 128(10):505-10.

21. Arshi Naz, Danish Zahid, Samna Naz, Muhammed Nadeem, Bijon Kumar, Tahir Sultan et al. Evaluvation of efficiency of varios immunochromatographic rapid tests for dengue diagnosis. Pak J Med Sci 2014;30(1):166-71.

22. Selvaraj Stephen, Marie Victor, Velmurugan Anitharaj, Civamany Deepa, Sivaraman et al. Early dengue diagnosis by non-structural protein 1 antigen detection: Rapid immunochromatography versus two enzyme-linked immunosorbent assay kits. Indian J Pathology Microbiol 2014;69(2):23-45.

\section{How to cite this article:}

Vidhya, V.R. and Palaniappan, N. 2019. Role of NS1 Antigen in Early Detection of Dengue during Epidemics. Int.J.Curr.Microbiol.App.Sci. 8(04): 229-236.

doi: https://doi.org/10.20546/ijcmas.2019.804.024 